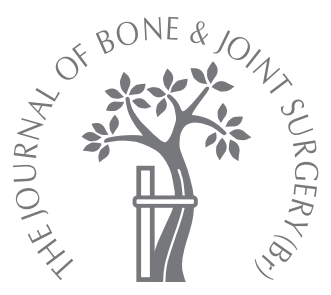
A. Gulati,
R. Chau,
H. G. Pandit,
H. Gray,
A. J. Price,
C. A. F. Dodd,
D. W. Murray
From Nuffield
Orthopaedic Centre
University of $\mathrm{O} x$ ford, Oxford, England

A. Gulati, MRCS, Clinica Research Fellow

H. G. Pandit, FRCS(Orth),

Senior Research Fellow

Botnar Research Centre

Nuffield Department of

Orthopaedic Surgery

In. Chau, MRCS, Clinical

Research Fellow

A. J. Price, FRCS(Orth),

Consultant Orthopaedic

Surgeon

C. A. F. Dodd, FRCS,

Consultant Orthopaedic

Surgeon

D. W. Murray, FRCS(Orth),

Professor and Consultant

Orthopaedic Surgeon

Nuffield Orthopaedic Centre

University of Oxford,

Headington, Oxford OX3 7LD,

UK.

H. Gray, DPhil, Senior

Lecturer

Department of Mechanical

Engineering

University of Moratuwa,

Katubedda, Moratuwa

Sri Lanka.

Correspondence should be sent to Professor D. W. Murray;

e-mail:

Barbara.Marks@ndorms.ox.ac.uk

(C)2009 British Editorial Society

of Bone and Joint Surgery

doi:10.1302/0301-620X.91B7.

$21914 \$ 2.00$

$J$ Bone Joint Surg [Br] 2009;91-B:896-902.

Received 14 October 2008;

Accepted after revision 19

March 2009

\title{
The incidence of physiological radiolucency following Oxford unicompartmental knee replacement and its relationship to outcome
}

\begin{abstract}
Narrow, well-defined radiolucent lines commonly observed at the bone-implant interface of unicompartmental knee replacement tibial components have been referred to as physiological radiolucencies. These should be distinguished from pathological radiolucencies, which are poorly defined, wide and progressive, and associated with loosening and infection. We studied the incidence and clinical significance of tibial radiolucent lines in 161 Oxford unicondylar knee replacements five years after surgery. All the radiographs were aligned with fluoroscopic control to obtain views parallel to the tibial tray to reveal the tibial bone-implant interface.

We found that 49 knees $(30 \%)$ had complete, $52(32 \%)$ had partial and $60(37 \%)$ had no radiolucent lines. There was no relationship between the incidence of radiolucent lines and patient factors such as gender, body mass index and activity, or operative factors including the status of the anterior cruciate ligament and residual varus deformity. Nor was any statistical relationship established between the presence of radiolucent lines and clinical outcome, particularly pain, assessed by the Oxford Knee score and the American Knee Society score.

We conclude that radiolucent lines are common after Oxford unicompartmental knee replacement but that their aetiology remains unclear. Radiolucent lines were not a source of adverse symptoms or pain. Therefore, when attempting to identify a source of postoperative pain after Oxford unicompartmental knee replacement the presence of a physiological radiolucency should be ignored.
\end{abstract}

The presence of periprosthetic radiolucencies following joint replacement is usually considered to be indicative or predictive of loosening. ${ }^{1} \mathrm{How}^{-}$ ever, a high incidence of radiolucent lines has been observed on post-operative radiographs of the Oxford unicompartmental knee replacement (UKR) (Biomet, Swindon, United Kingdom) without such an association. 2,3 Tibrewal, Grant and Goodfellow, ${ }^{4}$ in a study of 80 knees, found that the incidence of partial or complete tibial radiolucent lines was $96 \%$.

Goodfellow et $\mathrm{al}^{5}$ subdivided radiolucencies into two distinct subtypes. The most common is described as a physiological radiolucency or a radiolucent line. These develop during the first post-operative year and consolidate thereafter without progression. Generally they are about $1 \mathrm{~mm}$ thick, but can be up to $2 \mathrm{~mm}$. They are well defined and have parallel running radiodense lines. In contrast, the rarer type of radiolucency is pathological and is associated with loosening or infection. These are progressive, poorly defined, $>2 \mathrm{~mm}$ thick and have no matching radiodense line (Fig. 1).
The incidence of radiolucent lines depends primarily on how the radiographs are taken. Images obtained using an image intensifier to ensure the X-ray beam is aimed parallel to the interface will reveal radiolucent lines (Fig. 2). Conversely in standard radiographs, which are not taken parallel to the interface, radiolucent lines are usually not seen as they are obscured by the radio-opacity of the implant. ${ }^{6}$ With well-aligned screened radiographs, the majority of Oxford UKR tibial components have radiolucent lines at one year, yet at ten and 20 years the incidence of loosening is very low. ${ }^{7,8}$ This suggests that radiolucent lines are not associated with, nor predictive of, loosening. Despite this, faced with a patient reporting pain and with a radiolucent line, many surgeons will feel that the radiolucent line is the source of the pain and will convert these cases to a total knee replacement (TKR). In order to determine whether the cause of pain is related to radiolucent lines, a large study is necessary.

Despite radiolucent lines being very common, their aetiology is not known. Although a 


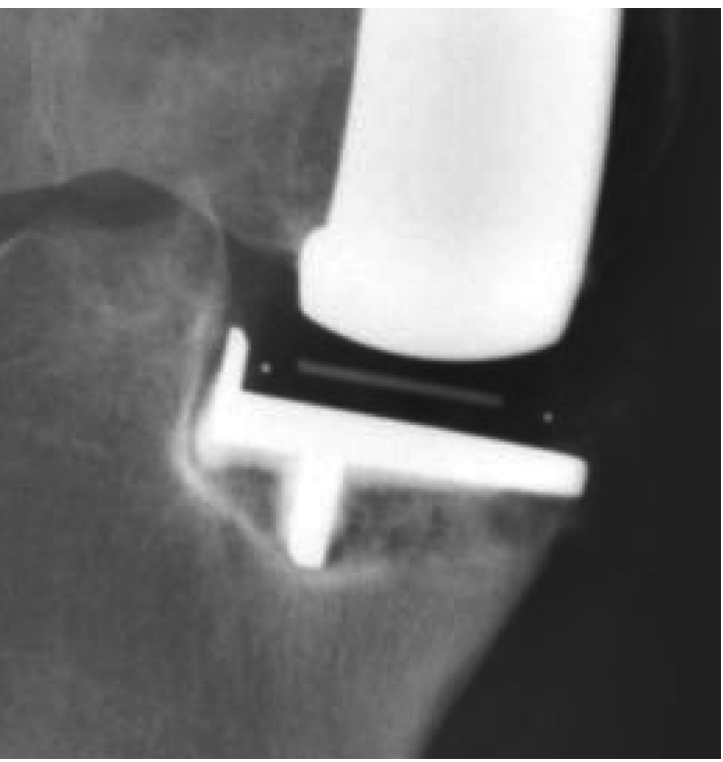

Fig. 1

Radiograph showing a pathological radiolucency beneath the tibial component of an Oxford UKR.

number of hypotheses have been proposed, ${ }^{9-12}$ none have been established. In order to gain insight into the aetiology of radiolucent lines, it is necessary to determine whether they are due to patient factors, such as body mass index (BMI) and activity level, or surgical factors, such as the state of the anterior cruciate ligament (ACL) or postoperative leg alignment.

The aims of this study were to establish the incidence, aetiology and clinical significance of radiolucent lines following the Oxford UKR. This was done in a cohort of patients who had radiographs accurately aligned with the tibial component five years after operation.

\section{Patients and Methods}

From our database we identified 161 knees in 138 patients who had undergone medial phase 3 Oxford UKR and satisfied the criteria for this study. All knees had the established indications for the Oxford UKR, ${ }^{5}$ with diagnoses of either anteromedial osteoarthritis $(\mathrm{n}=158)$ or avascular necrosis $(\mathrm{n}=3)$. All operations were carried out by two consultant surgeons (DM and CD), using a short incision without patellar dislocation. ${ }^{5}$ All knees had follow-up radiographs that were perfectly aligned with the tibial bone-implant interface at approximately five years. These strict criteria resulted in the exclusion of a number of patients; out of 211 medial UKRs with appropriate indications and five-year follow-up, only 161 had appropriate radiographs. The others either did not have radiographs for social or geographic reasons, or had films that were not perfectly aligned. Out of the whole group, no patients have been lost to follow-up and no knees have been revised for tibial loosening.

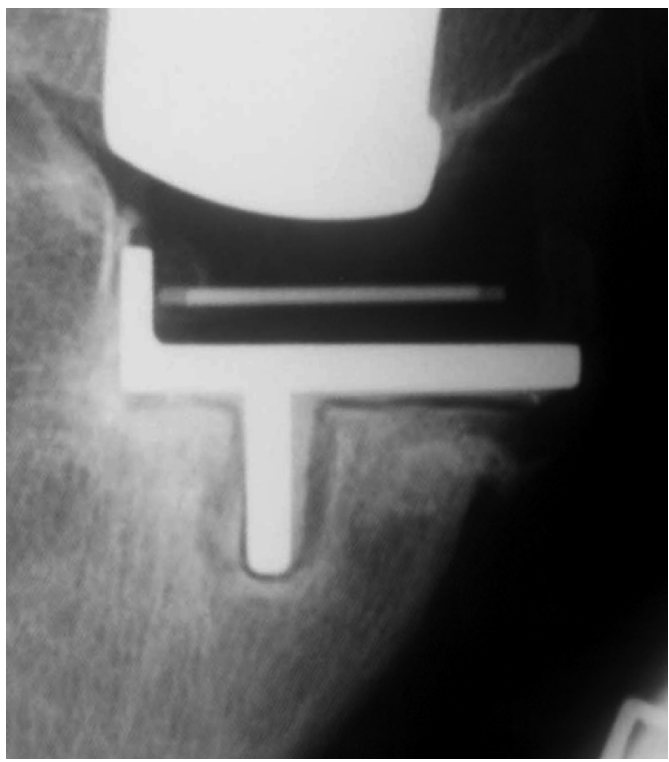

Fig. 2

Radiograph showing an extreme example of a physiological radiolucency beneath the tibial component of an Oxford UKR.

The patients were evaluated clinically and radioglogically before operation and after five years. Demographic and intra-operative data were also collected. The outcome measures were the Oxford knee score (OKS), ${ }^{13}$ the American Knee Society score (AKSS), ${ }^{14}$ Objective and Function (AKSS-O, AKSS-F), and the Tegner activity score (TS). ${ }^{15}$ The OKS ranges between 0 and 48, with 48 being the best. ${ }^{16}$ In addition, a pain score was deduced from the OKS and AKSS. For the OKS, this is the combined score from questions $1,4,5,8$ and 9, which all directly relate to the patient's perception of pain. ${ }^{17}$ The OKS pain score ranges from 0 to 20, with a score of 20 representing no pain. The AKSS pain score is the score from question 1 and ranges from 0 to 50 . Changes in the OKS and the OKS pain score $(\Delta \mathrm{OKS}$ and $\triangle \mathrm{PS}$ respectively), relative to the pre-operative value, were also calculated.

All the post-operative radiographic assessments were made under fluoroscopic guidance with the patient supine. For the anteroposterior (AP) view the direction of the beam was adjusted until the tibial plateau and vertical wall were as narrow as possible, thereby ensuring that the X-ray beam was parallel to the undersurface of the tibial component. The films were scanned at $300 \mathrm{dpi}$ resolution and converted to jpeg format (Epsom Expression 1640XL scanner with Transparency Unit, Epson, California) for evaluation. Correction for magnification was performed by comparing the apparent radius of curvature of the femoral component on the digital image with the known radius of the implanted component. The area under the tibial tray was divided into six regions (Fig. 3). In each the presence or absence of a radiolucent line was recorded. The thickness of the radiolucency was mea- 


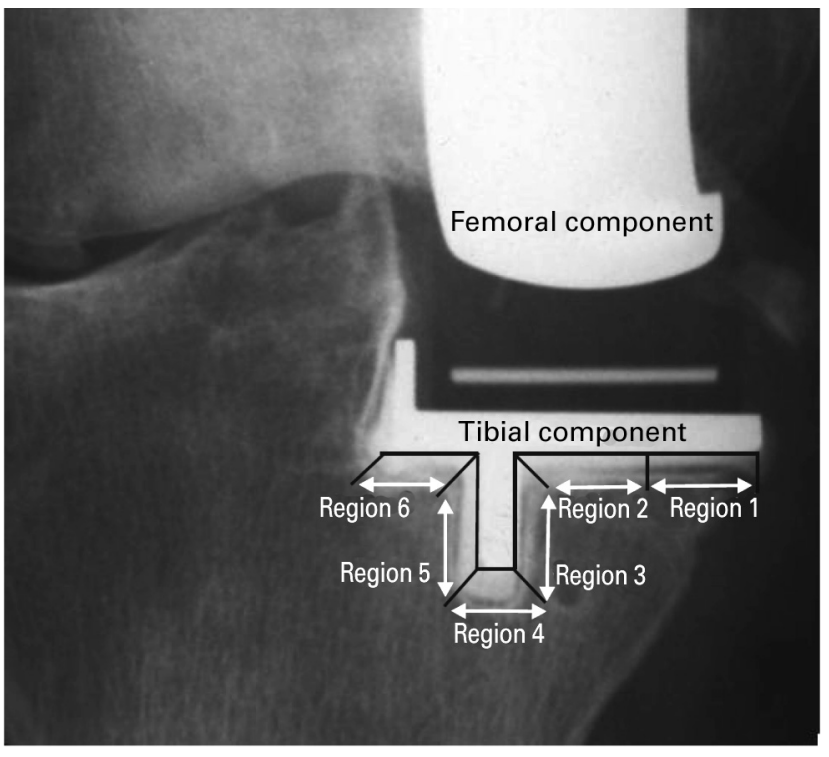

Fig. 3

Radiograph showing the six regions for the distribution of radiolucent lines beneath the tibial component of the Oxford UKR.

sured and the presence of a sclerotic radiodense margin noted. Radiolucencies beside the vertical wall were not assessed, as this is not a site where the component is fixed and no cement is placed there.

In order to determine which factors were related to radiolucent lines, their distribution in each patient was classified in three ways with presence or absence; complete (all six tibial regions having a radiolucent line), partial (1 to 5 regions) and none. The relationship between the categorisation and the following were then analysed:

1. Patient variables: age at the time of surgery, BMI, gender, pre-operative clinical scores (OKS, AKSS, TS) and postoperative activity level (TS).

2. Operative factors: ACL status, residual post-operative varus alignment and the operating consultant.

3. Outcome measures: five-year OKS, AKSS-O, AKSS-F, TS and the pain elements of the OKS and AKSS. Also the change in OKS and pain score from before operation to five years after $(\triangle \mathrm{OKS}, \Delta \mathrm{PS})$.

Statistical analysis. Analyses were carried out using SPSS for Windows v 12.0 (SPSS Inc., Chicago, Illinois). For continuous data, Student's $t$-test was used for two groups of variables and one-way analysis of variance (ANOVA) for more than two groups, followed by post hoc Tukey analysis. For categorical data, Pearson's chi-squared test was used. Statistical significance was denoted by $\mathrm{p}<0.05$. A power calculation ${ }^{18}$ indicated that 120 knees would be required to detect a difference in pain score of two points between the groups.

The measurements in this study were made by one researcher $(\mathrm{RC})$ and were checked by another $(\mathrm{HP})$. Neither had performed the surgery, and the radiographic analysis was conducted blinded to the clinical scores. In order to assess intra- and inter-observer variability, repeated measurements in a subgroup of patients were made by two researchers (RC, AG). The correlation coefficients for both intra- and inter-observer data were $>0.98(\mathrm{p}<0.01)$.

\section{Results}

Of the 161 Oxford UKRs in the study, 101 (63\%) were found to have radiolucent lines under the tibial component on radiographs five years after operation. In 49 knees $(30 \%)$ these involved all six zones, $52(32 \%)$ had partial and $60(37 \%)$ had no radiolucent lines. None of the radiolucencies was greater than $1 \mathrm{~mm}$ thick, and all had a sclerotic margin. They were therefore all considered to be physiological.

The demographics of the patients in the radiolucent and non-radiolucent line groups were comparable (Table I). The groups were well matched and there was no statistically significant difference in the pre-operative criteria; the mean age at surgery, the mean BMI or gender, between the radiolucent and non-radiolucent line group. There was no statistical difference between the two groups in the operating surgeon, status of the ACL, or the degree of post-operative residual varus alignment of the leg (Table II). The differences in clinical scores, including the pain scores, between the groups were also not statistically significant (Table I).

When considering the presence of no, partial or complete radiolucent lines, no statistical significance was found in all the variables between groups (Table III).

The knees were divided into groups according to the number of regions with radiolucent lines, but this revealed no statistically significant difference in any variable between the groups. Partial radiolucent lines were most commonly seen under the flat surface of the tibial component rather than around the keel (Fig. 4) $(p=0.014)$. The mean width of the radiolucent lines was $0.73 \mathrm{~mm}(0.1$ to $1.0)$ and there was no significant difference in the width of radiolucent lines seen in the different regions $(p=0.234)$.

\section{Discussion}

This study confirms that radiolucent lines commonly occur under the tibial component of the Oxford UKR. We found that approximately one-third of cases had complete radiolucent lines, one-third had partial and one-third had none. Tibrewal et $\mathrm{al}^{4}$ were the first to report a high incidence of radiolucent lines with the Oxford knee replacement, but in a series of patients who had undergone bicompartmental rather than unicompartmental procedures. In that study, the high incidence was attributed primarily to the fluoroscopically aligned radiographic technique used for the postoperative assessment of the implant. We also routinely use fluoroscopically aligned radiographs following Oxford UKR and are able to confirm that with accurately aligned radiographs, a high incidence of radiolucent lines will be identified. We have also observed that radiolucent lines seen during screening will be concealed if the fluoroscope is tilted by just $2^{\circ}$ (Fig. 5). In order to detect radiolucencies it is necessary 
Table I. Demographic information and scores with means presented with standard deviation (SD) in the two groups; no radiolucent lines (no RLLs) and radiolucent lines (RLLs)

\begin{tabular}{|c|c|c|c|}
\hline & No RLLs ( $n=60,37.3 \%$ ) & RLLs ( $n=101,62.7 \%$ ) & p-value \\
\hline Age (yrs) & $66.5(8.7)$ & $66.2(9.0)$ & 0.856 \\
\hline \multicolumn{4}{|l|}{ Gender } \\
\hline Female & 36 & 52 & 0.289 \\
\hline Male & 24 & 49 & \\
\hline Body mass index & $27.9(4.9)$ & $27.5(3.8)$ & 0.580 \\
\hline \multicolumn{4}{|l|}{ Pre-operative scores ${ }^{*}$} \\
\hline OKS & $19(8)$ & $19(8)$ & 0.789 \\
\hline AKSS-O & $36(6)$ & 34 (9) & 0.434 \\
\hline AKSS-F & $50(12)$ & $51(10)$ & 0.941 \\
\hline Tegner & $2(1)$ & $2(0)$ & 0.994 \\
\hline \multicolumn{4}{|c|}{ Five-year follow-up scores } \\
\hline OKS & $40(9)$ & $40(8)$ & 0.849 \\
\hline AKSS-O & $88(14)$ & $89(13)$ & 0.736 \\
\hline AKSS-F & 87 (17) & $85(18)$ & 0.695 \\
\hline Tegner & $3(1)$ & $3(1)$ & 0.636 \\
\hline \multicolumn{4}{|l|}{ Pain scores } \\
\hline OKS Q1, 4, 5, 8, 9 & $17(4)$ & $17(4)$ & 0.428 \\
\hline AKSS Q1 & $44(10)$ & $45(9)$ & 0.370 \\
\hline \multicolumn{4}{|l|}{5 -year change in scores } \\
\hline$\Delta \mathrm{OKS}^{\dagger}$ & $21(11)$ & $22(10)$ & 0.906 \\
\hline \multicolumn{4}{|l|}{ Pain scores } \\
\hline OKS Q1, 4, 5, 8, 9 & $9(5)$ & $11(4)$ & 0.210 \\
\hline
\end{tabular}

Table II. Degree of anterior cruciate ligament (ACL) damage and alignment in the three groups (no radiolucent lines ( $R L L$, ) partial $R L L$, and complete $R L L$ )

\begin{tabular}{lrrr}
\hline & No RLL & Partial RLL & Complete RLL \\
\hline ACL grade (chi-squared $\mathrm{p}=0.473)$ & & & \\
1. Normal & 47 & 39 & 39 \\
2. Synovial damage & 8 & 10 & 4 \\
3. Longitudinal splits & 4 & 3 & 6 \\
4. Friable and fragmented & 1 & 0 & 0 \\
5. Absent & 0 & 0 & 0 \\
& & & \\
Valgus/varus alignment (chi-squared $\mathrm{p}=0.921)$ & & & 3 \\
1. Varus $<0^{\circ}$ & 4 & 4 & 14 \\
2. Valgus $\left(0^{\circ}\right.$ to $\left.4^{\circ}\right)$ & 17 & 10 & 32 \\
3. Normal $\left(5^{\circ}\right.$ to $\left.10^{\circ}\right)$ & 39 & 36 & 0 \\
4. Valgus $\left(>10^{\circ}\right)$ & 0 & 2 & \\
\hline
\end{tabular}

not only to use a fluorscope but also to ensure the X-ray beam is accurately aligned. Standard radiographs or poorly aligned screened radiographs may not detect radiolucencies.

In our series the patient-related factors, $\mathrm{BMI}^{19}$ and activity level, ${ }^{20}$ which influence the load transmitted by the knee and which might influence loosening of UKR, had no statistically significant relationship with the presence of radio- lucent lines. Similarly, surgical factors such as residual varus deformity ${ }^{21}$ or ACL damage ${ }^{22}$ that might also increase loosening had no statistical relationship to the incidence of radiolucent lines. It therefore remains unclear what factors, if any, are likely to lead to their formation.

Many theories have been proposed to explain the aetiology of radiolucent lines. Thermal necrosis due to the heat of 
Table III. Demographic information and mean scores (standard deviation) in the three groups; no radiolucent lines (RLL), partial RLLs and complete RLLs

\begin{tabular}{|c|c|c|c|c|}
\hline & No RLL $(n=60)$ & Partial RLL (n = 52) & Complete RLL (n = 49) & p-value \\
\hline Age (yrs) & $66.5(8.7)$ & $66.5(8.8)$ & $66.0(9.3)$ & 0.935 \\
\hline \multicolumn{5}{|l|}{ Gender } \\
\hline Female & 36 & 28 & 24 & 0.528 \\
\hline Male & 24 & 24 & 25 & \\
\hline Body mass index & 27.9 (4.9) & $27.0(3.2)$ & $28.0(4.2)$ & 0.425 \\
\hline \multicolumn{5}{|l|}{ Pre-operative scores ${ }^{*}$} \\
\hline OKS & $19(8)$ & $20(9)$ & $17(7)$ & 0.139 \\
\hline AKSS-O & $36(6)$ & $36(10)$ & $33(9)$ & 0.400 \\
\hline AKSS-F & $50(12)$ & $52(10)$ & $49(10)$ & 0.658 \\
\hline Tegner & $2(1)$ & $2(1)$ & $1(1)$ & 1.000 \\
\hline \multicolumn{5}{|c|}{ Five-year follow-up scores } \\
\hline OKS & $40(9)$ & 41 (7) & $39(10)$ & 0.395 \\
\hline AKSS-O & $88(14)$ & $91(12)$ & $88(15)$ & 0.537 \\
\hline AKSS-F & 87 (17) & $87(15)$ & $84(21)$ & 0.700 \\
\hline Tegner & $3(1)$ & $3(1)$ & $3(1)$ & 0.884 \\
\hline \multicolumn{5}{|l|}{ Pain scores } \\
\hline OKS Q1, 4, 5, 8, 9 & $17(4)$ & $17(3)$ & $16(4)$ & 0.496 \\
\hline AKSS Q1 & $44(10)$ & $47(6)$ & $44(11)$ & 0.208 \\
\hline \multicolumn{5}{|c|}{ Five-year change in scores } \\
\hline$\Delta \mathrm{OKS}^{\dagger}$ & $21(11)$ & $20(9)$ & $23(8)$ & 0.533 \\
\hline \multicolumn{5}{|l|}{ Pain scores } \\
\hline$\triangle \mathrm{OKS} \mathrm{Q1,} \mathrm{4,} \mathrm{5,} \mathrm{8,} 9$ & $9(5)$ & $10(5)$ & $11(4)$ & 0.432 \\
\hline
\end{tabular}

polymerisation of cement and the resulting bone resorption has been suggested, ${ }^{9}$ although some ${ }^{23}$ but not all ${ }^{24}$ studies, have reported a higher incidence of tibial radiolucent lines in cementless than in cemented UKRs. ${ }^{23}$ There is some evidence that pulsed lavage may reduce the incidence of the former, suggesting that the cementing technique may influence their occurrence. ${ }^{10}$ The sequence of micromovement, local osteonecrosis and fibrocartilage tissue formation has also been proposed. However, Rea et $\mathrm{al}^{11}$ found no correlation between radiolucent lines and micromovement of the tibial component using radiostereometric analysis despite having a $75 \%$ incidence of radiolucent lines. It has been suggested that they form in response to compressive loading. ${ }^{12}$ With the Oxford UKR the mobile polyethylene insert minimises shearing forces through the tibial tray, so that most loads transmitted to the main tibial bone-cement interface, where radiolucent lines are most commonly seen, are compressive. Around the keel, where shearing force persists, they are less commonly seen. However radiolucent lines are seen around TKR and fixed-bearing UKR when the loads are not all compressive. $\mathrm{Li}$ et $\mathrm{a}^{25}$ have reported a lower incidence of radiolucent lines in mobile-bearing UKR than in fixed. Therefore the relationship between loading and radiolucency is complex.

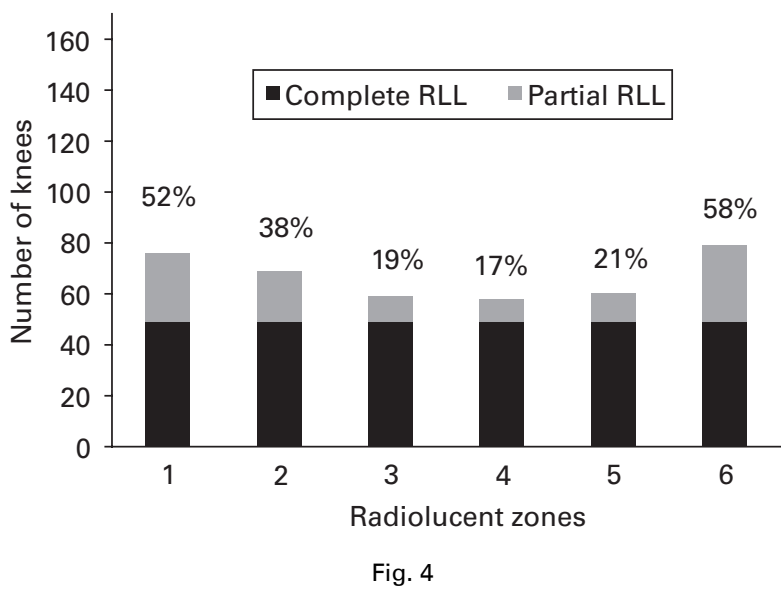

Bar chart showing distribution of physiological radiolucency in six zones. The figures represent the percentage of knees that have partial radiolucent lines (RLLs) in each zone.

Clinically the most important finding of this study was the absence of any statistically significant relationship between the presence and extent of the radiolucent lines 


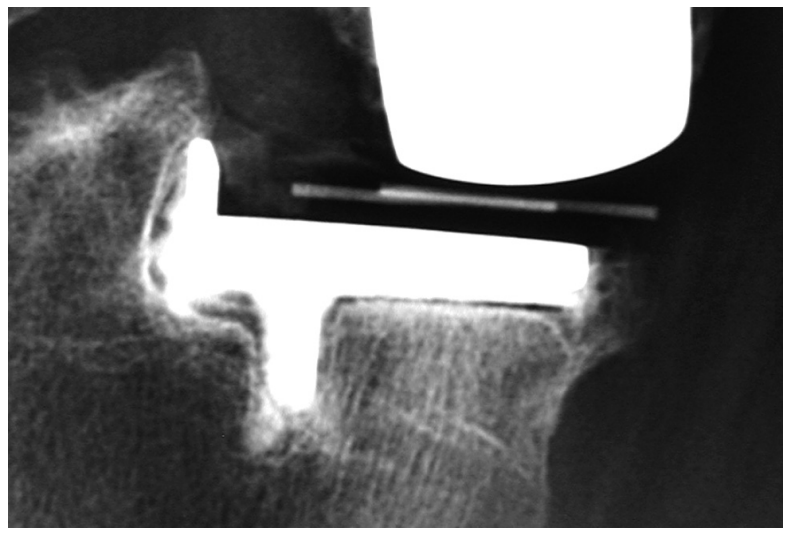

Fig. $5 a$

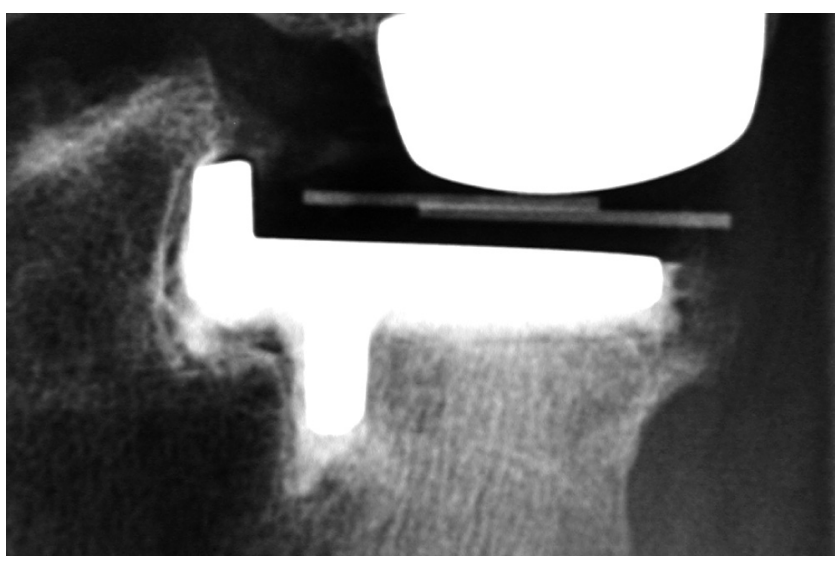

Fig. $5 b$

Radiographs a) perfectly aligned showing a radiolucent line and b) of the same implant taken with a $2^{\circ}$ tilt of the image intensifer, which has obscured the radiolucency.

and outcome. In particular, patients with complete radiolucent lines did not have more pain than those without. This suggests that the underlying process responsible for the formation of radiolucent lines does not cause pain. Therefore, if a patient reports pain following UKR and has a radiolucent line visible on a radiograph, it should not be assumed that the source of pain is the radiolucency.

We recognise the limitations of this study. Our findings with the Oxford UKR probably cannot be extrapolated to fixed-bearing UKR or TKR, as the interfacial loading is very different. Although the numbers of replacements were relatively large, a power calculation suggested that the study would not be able to detect an association between pain and radiolucent lines if the pain score difference was less than two points. Additionally, not all our patients could be included in the study, as some did not have adequate radiographs. It is therefore possible that those patients excluded had pain in association with radiolucent lines. However, this seems unlikely, as there were no revisions for tibial loosening in the whole group.
This study demonstrated that beneath the tibial component of the Oxford UKR, complete radiolucent lines were seen in one-third of patients, partial lines in another third, and only one-third had none. The frequency of their formation was not related to patient or surgical factors. It remains unclear why they occur. No relationship was established between radiolucent lines and outcome, particularly pain, suggesting that radiolucent lines are not a source of pain.

The authors wish to thank Mrs B. Marks, Ms C. Jenkins, Ms J. Copp and Ms J. Ferris for their assistance in undertaking this study. Financial support has been received from the NIHR Biomedical Research Unit into Musculoskeletal Disease, Nuffield Orthopaedic Centre and University of Oxford.

The author or one or more of the authors have received or will receive benefits for personal or professional use from a commercial party related directly or indirectly to the subject of this article. In addition, benefits have been directed to a research fund with which the authors are associated.

\section{References}

1. Ritter MA, Zhou H, Keating CM, et al. Radiological factors influencing femoral and acetabular failure in cemented Charnley total hip arthroplasties. J Bone Joint Surg [Br] 1999:81-B:982-6

2. Klemme WR, Galvin EG, Petersen SA. Unicompartmental knee arthroplasty: sequential radiographic and scintigraphic imaging with an average five-year follow-up. Clin Orthop 1994;301:233-8.

3. Berger RA, Nedeff DD, Barden RM, et al. Unicompartmental knee arthroplasty: clinical experience at 6- to 10-year followup. Clin Orthop 1999;367:50-60.

4. Tibrewal SB, Grant KA, Goodfellow JW. The radiolucent line beneath the tibial components of the Oxford meniscal knee. J Bone Joint Surg [Br] 1984;66-B:523-8.

5. Goodfellow JW, O'Connor JJ, Dodd CAF, Murray DW. Unicompartmental arthroplasty with the Oxford knee. Oxford: Oxford University Press, 2006:117-28.

6. Lundberg-Jensen A, Kourakis AH, Rossen B, Kofoed H. Radiolucency adjacent to the tibial component in total knee arthroplasty. Orthopedics 2002;25:49-51.

7. Murray DW, Goodfellow JW, 0'Connor JJ. The Oxford medial unicompartmental arthroplasty: a ten-year survival study. J Bone Joint Surg [Br] 1998;80-B:983-9.

8. Price AJ, Waite JC, Svard U. Long-term clinical results of the medial Oxford unicompartmental knee arthroplasty. Clin Orthop 2005;435:171-80.

9. Andersson GB, Freeman MA, Swanson SA. Loosening of the cemented acetabular cup in total hip replacement. J Bone Joint Surg [Br] 1972;54-B:590-9.

10. Aldinger PR, Hauck C, Clarius $\mathbf{M}$. The radiolucent line under the Oxford uni tibial tray -pulsed lavage versus conventional irrigation: a radiographic evaluation at min. 1 year postoperatively. Procs European Federation of National Association of Orthopaedics and Traumatology 2007.

11. Rea P, Short A, Pandit $\mathbf{H}$, et al. Radiolucency and migration after Oxford unicompartmental knee arthroplasty. Orthopedics 2007;30(Suppl):24-7.

12. O'Connor J, Goodfellow J, Perry N. Fixation of the tibial components of the Oxford knee. Orthop Clin North Am 1982;13:65-87.

13. Dawson J, Fitzpatrick R, Murray D, Carr A. Questionnaire on the perceptions of patients about total knee replacement. J Bone Joint Surg [Br] 1998;80-B:63-9.

14. Insall JN, Dorr LD, Scott RD, Scott WN. Rationale of the Knee Society clinical rating system. Clin Orthop 1989;248:13-14

15. Tegner Y, Lysholm J. Rating systems in the evaluation of knee ligament injuries. Clin Orthop 1985;198:43-9.

16. Murray DW, Fitzpatrick R, Rogers K, et al. The use of the Oxford hip and knee scores. J Bone Joint Surg [Br] 2007;89-B:1010-14.

17. Weale AW, Murray DW, Baines J, Newman JH. Radiological changes five years after unicompartmental knee replacement. J Bone Joint Surg [Br] 2000;82-B:996-1000.

18. Altman DG. Statistics and ethics in medical research. III: how large a sample? Br Med J 1980;281:1336-8

19. Berend KR, Lombardi AV Jr, Adams JB. Obesity, young age, patellofemoral disease, and anterior knee pain: identifying the unicondylar arthroplasty patient in the United States. Orthopedics 2007;30(Suppl):19-23.

20. Schai PA, Suh JT, Thornhill TS, Scott RD. Unicompartmental knee arthroplasty in middle-aged patients: a 2- to 6-year follow-up evaluation. J Arthroplasty 1998;13:365-72.

21. Hernigou $\mathbf{P}$, Deschamps G. Alignment influences wear in the knee after medial unicompartmental arthroplasty. Clin Orthop 2004;423:161-5. 
22. Goodfellow JW, Kershaw CJ, Benson MK, O'Connor JJ. The Oxford Knee for unicompartmental osteoarthritis: the first 103 cases. J Bone Joint Surg [Br] 1988;70B:692-701.

23. Forsythe ME, Englund RE, Leighton RK. Unicondylar knee arthroplasty: a cementless perspective. Can J Surg 2000;43:417-24.
24. Pandit H, Jenkins C, Beard DJ, et al. Cementless Oxford unicompartmental knee replacement shows reduced radiolucency at one year. J Bone Joint Surg [Br] 2009:91B:185-9.

25. Li MG, Yao F, Joss B, et al. Mobile vs fixed bearing unicondylar knee arthroplasty: a randomized study on short term clinical outcomes and knee kinematics. Knee2006;13:365-70. 